\title{
A survey of flux transfer events observed by Cluster during strongly northward IMF
}

\author{
R. C. Fear, A. N. Fazakerley, and C. J. Owen \\ Mullard Space Science Laboratory, University College London, Dorking, UK \\ E. A. Lucek \\ Blackett Laboratory, Imperial College London, London, UK
}

Received 14 June 2005; revised 11 August 2005; accepted 18 August 2005; published 20 September 2005.

[1] During Cluster's annual dayside seasons (NovemberJune) the four spacecraft cross the magnetopause at high latitudes near local noon, and at lower latitudes further along the flanks. During these crossings, observations of flux transfer events (FTEs), a signature of transient or variable-rate magnetopause merging, are often made. We have compiled a survey of FTEs observed by Cluster in the 2002/3 dayside season. A significant number of FTEs, presented here, were observed under strongly northward IMF. Multi-spacecraft techniques enable more accurate velocities to be calculated than previously possible. The observed velocities are consistent with a long, component merging X-line emanating from the antiparallel merging site in the lobe, but require a relaxation of the antiparallel merging hypothesis to allow the X-line to extend to regions of lower shear on the flank. The velocities observed at lower latitudes are not consistent with a subsolar X-line. Citation: Fear, R. C., A. N. Fazakerley, C. J. Owen, and E. A. Lucek (2005), A survey of flux transfer events observed by Cluster during strongly northward IMF, Geophys. Res. Lett., 32, L18105, doi:10.1029/2005GL023811.

\section{Introduction}

[2] The magnetic merging process proposed by Dungey [1961] is widely regarded as the major means of transferring solar wind mass and momentum into the Earth's magnetosphere under southward IMF. FTEs, first reported by Russell and Elphic [1978], are now generally accepted to be consequences of transient magnetic merging. When viewed in the boundary normal coordinate frame introduced by Russell and Elphic [1978], FTEs can be recognized by a characteristic bipolar variation in the magnetic field component normal to the magnetopause $(\hat{\mathbf{N}})$. The polarity of the bipolar signature is determined by the motion of the FTE relative to the local, undisturbed magnetic field, which drapes around the FTE. In the magnetosheath, a 'standard' polarity signature in $\mathrm{B}_{N}(+/-$ relative to $\hat{\mathbf{N}}$, which is directed away from the Earth) is observed if the FTE velocity has a component antiparallel to the local magnetosheath magnetic field, whereas a 'reverse' signature $(-/+)$ occurs if the FTE velocity has a component parallel to the local field. Within the magnetosphere, a standard signature is observed when the velocity has a component parallel to the geomagnetic field, and a reverse signature when antiparallel. Since FTEs

Copyright 2005 by the American Geophysical Union. 0094-8276/05/2005GL023811 are generated in regions of high magnetic shear, the polarities observed by two nearby spacecraft on either side of the magnetopause are the same. These signatures are usually accompanied by an enhancement in the magnitude of the magnetic field [Paschmann et al., 1982], but occasionally a decrease is observed [Rijnbeek et al., 1984]. A mixture of plasma populations from either side of the magnetopause will be observed if the spacecraft crosses recently reconnected field lines [Daly et al., 1981].

[3] Several statistical studies have shown that FTEs occur predominantly when IMF $\mathrm{B}_{Z}<0$ [Kuo et al., 1995, and references therein], although these surveys were restricted to observations in the pre-terminator region $\left(\mathrm{X}_{G S M}>0\right)$. Standard polarity FTEs are generally observed in the Northern Hemisphere, and reverse events in the Southern Hemisphere, consistent with FTEs being generated by dayside low-latitude merging (i.e. equatorward of the cusps). Kawano and Russell [1997a] have shown FTE polarity, and hence motion, to be consistent with lowlatitude merging even when there is a dominant IMF $\mathrm{B}_{Y}$ component. This is the major piece of evidence for the component merging model [Gonzalez and Mozer, 1974], as opposed to strictly antiparallel merging [Crooker, 1979].

[4] High-latitude merging may occur between magnetosheath and lobe magnetic fields when $\mathrm{B}_{Z}>0$ [Dungey, 1963]. Freeman et al. [1993] suggested that subsolar merging occurs for IMF clock angles $\left|\theta_{C A}\right|>70^{\circ}$, and lobe merging occurs when $\left|\theta_{C A}\right|<70^{\circ}\left(\theta_{C A}=\arctan \left[B_{Y} / B_{Z}\right]\right.$, where $\mathrm{B}_{Y}$ and $\mathrm{B}_{Z}$ are GSM components of the IMF). Observations of FTEs when IMF $\mathrm{B}_{Z}>0$ are few, but Kawano and Russell [1997b] examined 144 FTEs in the post-terminator region $\left(\mathrm{X}_{G S M}<0,\left|\mathrm{Z}_{G S M}\right|<15\right), 79$ of which occurred when $\mathrm{B}_{Z}>0$. They concluded from the polarities of the signatures that a tilted equatorial merging line (X-line), could explain most events when $\left|\theta_{C A}\right|<90^{\circ}$ if flux tubes in the subsolar region were prevented from being observed by 're-reconnection' [Nishida, 1989]. When the IMF was more strongly northward, the polarities and $\mathrm{B}_{Y}$ dependency could also be explained by the FTEs being generated near the polar cusp at an antiparallel merging site, then moving equatorward and tailward.

[5] We have carried out a survey of FTEs observed over one season of Cluster dayside magnetopause crossings (November 2002-June 2003). Here we present the statistics of the events which were observed under strongly northward IMF conditions, which we define as $\left|\theta_{C A}\right|<70^{\circ}$. Fourspacecraft timing analysis enables the determination of FTE velocities, which we compare with a model. 


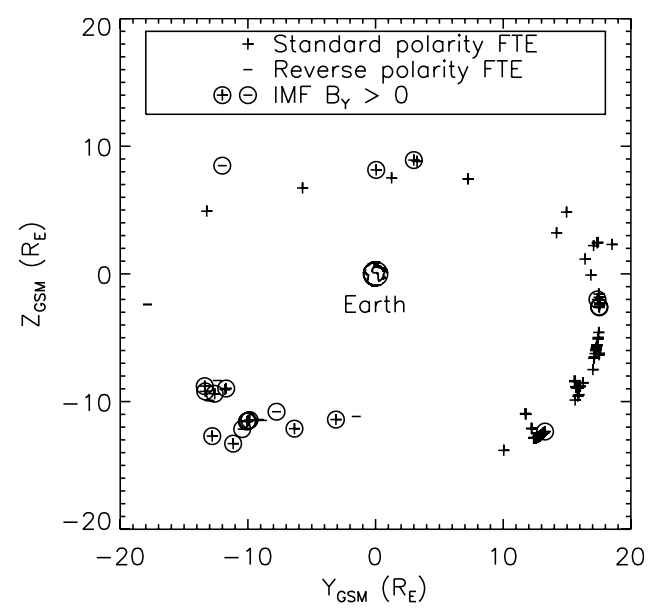

Figure 1. The location of all FTEs which occurred when the lagged IMF was within $\pm 70^{\circ}$ of north. The location of each FTE is projected into the GSM Y-Z plane. The plot symbol i.e., +/- denotes the FTE polarity, and FTEs which occurred with a lagged IMF $\mathrm{B}_{Y}>0$ are encircled.

\section{Methodology and FTE Selection Criteria}

[6] The 2002/3 dayside season was selected as the separation of the four spacecraft in the vicinity of the magnetopause was comparable to the scale size of an FTE $(\sim 5000 \mathrm{~km})$ for most of the season, enabling determination of FTE motion from multi-spacecraft techniques. Six-hour periods of data from the Flux Gate Magnetometer (FGM) [Balogh et al., 2001], centered on each observed magnetopause crossing time, were examined in detail along with data from the Plasma Electron and Current Experiment where available (PEACE) [Johnstone et al., 1997]. The duration of the inspected interval was extended for crossings in the flank regions, when Cluster skimmed the magnetopause for up to 18 hours. The magnetic field data were transformed into the boundary normal coordinate frame introduced by Russell and Elphic [1978] using a magnetopause normal derived from the Roelof and Sibeck [1993] model. Minimum variance analysis (MVA) was also carried out on each crossing.

[7] The following events were selected:

[8] 1. A bipolar variation that was clear in relation to the background variation of the magnetic field was required in either $\mathrm{B}_{N}$ (where $\hat{\mathbf{N}}$ was derived from the model) or the minimum variance component of $\mathbf{B}$ (when MVA on the magnetopause crossing was successful).

[9] 2. An enhancement or decrease was also required in $|\mathbf{B}|$, which had to be centered close to the center of the $\mathrm{B}_{N}$ signature.

[10] 3. Bipolar signatures centered on magnetopause crossings were excluded unless they were observed by at least one other spacecraft which did not cross the magnetopause. This criterion was chosen to reduce the possibility of FTE-like signals being caused by transient motion of the magnetopause across the spacecraft [e.g., Kuo et al., 1995].

[11] 446 FTEs were identified which satisfied these criteria on one or more spacecraft. 286 FTEs were observed only in the magnetosheath, and 50 in the magnetosphereproper. A further 32 were observed only in a boundary layer. A boundary layer was defined as having both a distinct electron distribution from the magnetosheath/ magnetosphere-proper (where PEACE data were available), and a magnetic field orientation that was also different from either side or which was similar to the magnetosphere-proper but more turbulent. 78 events were observed when the tetrahedron straddled two of these regions.

\section{Data Analysis}

[12] ACE data were available for 421 of the 446 FTEs. The appropriate IMF lag was deduced for each FTE by evaluating the time of arrival of each 4-minute parcel of solar wind plasma in the three hours beforehand, using $\mathrm{V}_{X G S M}$ observed at $\mathrm{ACE}$ and the separation in $\mathrm{X}_{G S M}$ of $\mathrm{ACE}$ and Cluster 3. The lagged IMF was then plotted with the magnetosheath field observed by Cluster, and the lag was adjusted by eye where necessary.

[13] 120 FTEs were observed under strongly northward IMF conditions, 99 of which occurred at $\mathrm{X}_{G S M}<0$. The locations and polarities of all FTEs which occurred when $\left|\theta_{C A}\right|<70^{\circ}$ are shown in Figure 1. The location of Cluster 3 at the time of each FTE observation has been projected into the GSM Y-Z plane. Of these 120 events, 90 were observed only in the magnetosheath, 13 in a boundary layer, 6 in the magnetosphere-proper and 11 when the tetrahedron straddled two of these regions. Events occurring under duskward IMF were mostly observed on the dawn flank, and vice versa. The FTEs were predominantly standard polarity, although 11 reverse events were observed.

[14] Multi-spacecraft timing analysis [Harvey, 1998] was applied to the 51 strongly northward IMF FTEs which were observed with a clear bipolar $\mathrm{B}_{N}$ signature on all four spacecraft prior to 10 June 2003, when an orbital manoeuvre rearranged the Cluster quartet into two pairs. The time difference was determined by maximizing the cross-correlation function between the $\mathrm{B}_{N}$ signature observed by Cluster 3 and each of the other three spacecraft. The velocities (V) are shown in Figure 2. The majority of the 51 events occurred on the dusk flank; most occurred in the post-terminator region and in the Southern Hemisphere. All of the dusk-flank FTEs moved antisunward; most which

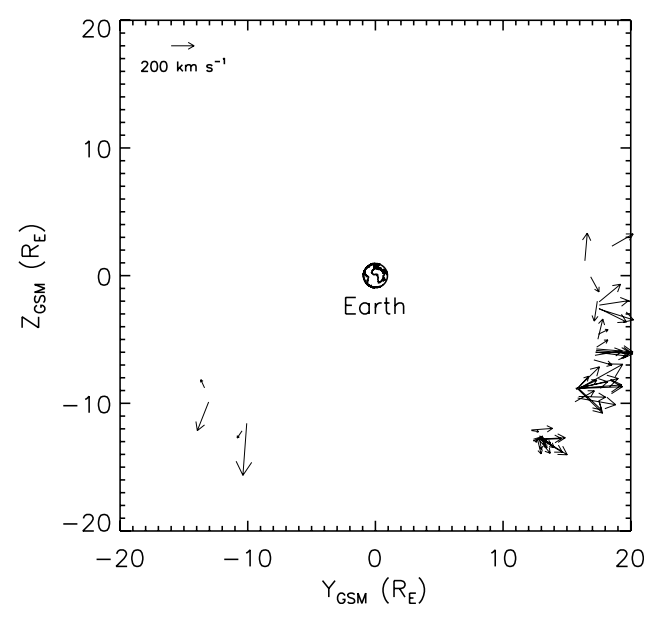

Figure 2. FTE velocities calculated by multi-spacecraft timing analysis, projected into the GSM Y-Z plane. The length of the arrow is proportional to the magnitude of the velocity within the Y-Z plane. 


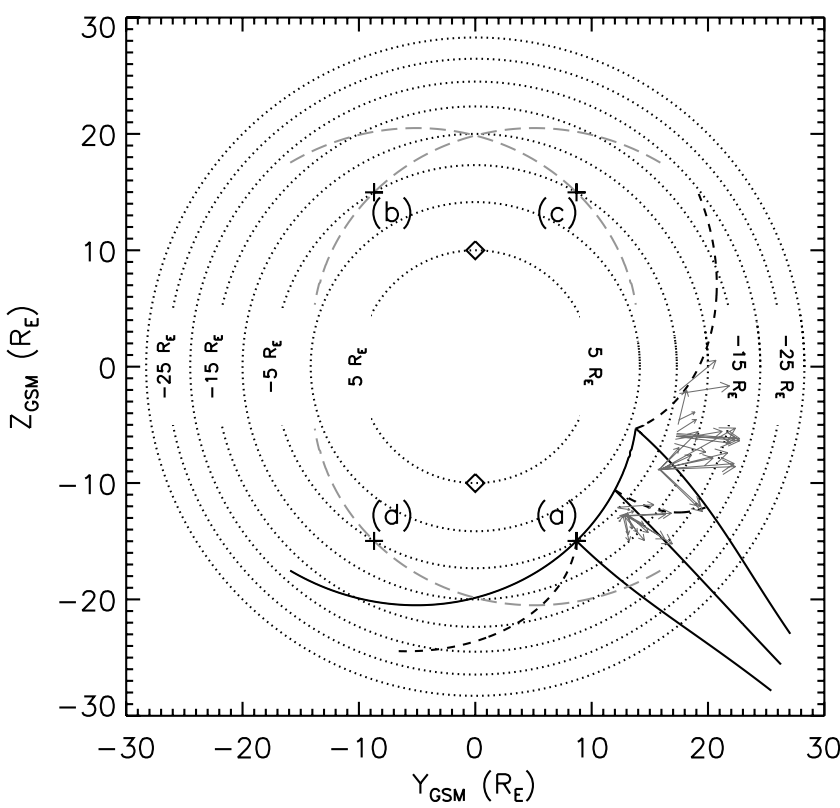

Figure 3. Evolution of flux tubes predicted by the model of Cooling et al. [2001]. The figure shows a view in the GSM Y-Z plane of the magnetopause. Concentric circles represent contours of $\mathrm{X}_{G S M}$. The diamonds are the positions of the cusps. Solid and dashed black lines denote model flux tube paths, and grey arrows show the location and velocities of the FTEs observed on the 10, 12 and 17 November. See text for more details.

occurred at $\mathrm{Z}_{G S M}<-10 \mathrm{R}_{E}$ had a negative $\mathrm{V}_{Z}$ (13 out of 17 FTEs), but 17 of the 30 FTEs at $Z_{G S M}>-10 \mathrm{R}_{E}$ had a small, positive $\mathrm{V}_{Z}$ component. Few velocities were determined on the dawn flank, so it is difficult to draw generalizations about the motion of these FTEs. However, the behavior of the examples we do have appears to be similar to that observed on the dusk flank; there were three southward-moving FTEs where $\mathrm{Z}_{G S M}<-10 \mathrm{R}_{E}$, and one lower-latitude FTE which moved equatorward.

\section{Comparison With Model}

[15] The model developed by Cooling et al. [2001] calculates the motion of reconnected flux tubes over the surface of a model magnetopause for specified magnetosheath and solar wind conditions. Flux tube motion is based on a simple model of the geomagnetic field $\left(\mathbf{B}_{g m}\right)$, and a draped magnetosheath field model $\left(\mathbf{B}_{m s}\right)$. The X-line is traced perpendicular to $\left(\mathbf{B}_{m s}-\mathbf{B}_{g m}\right)$ from a user-specified initial merging site. We conducted runs of this model using typical conditions from the 10,12 and 17 November, when the majority of our events occurred. The result of the 17 November run is shown in Figure 3. The mean of the IMF vector at the time of each FTE observation on this day was used $\left[(2,-7,7)_{G S M} \mathrm{nT}\right]$, along with a solar wind speed of $480 \mathrm{~km} \mathrm{~s}^{-1}$ and a solar wind plasma density of $8 \mathrm{~cm}^{-3}$, consistent with values observed by ACE. Model runs for the 10 and 12 November showed similar characteristics, as the mean IMF vectors were $(2,-7,12)$ and $(8,-7,5) \mathrm{nT}$ respectively.

[16] Two regions of high magnetic shear across the magnetopause are produced by the resulting draped magnetosheath magnetic field. This results in two possible $\mathrm{X}$-lines, marked in Figure 3: one in the Southern Hemisphere (a) and one in the Northern Hemisphere (b). If merging occurs at (b) the model requires a lower solar wind density than observed $\left(\sim 1 \mathrm{~cm}^{-3}\right)$ for the FTEs to be observed by Cluster, as this results in a sub-Alfvénic magnetosheath flow, which prevents the equatorward flux tubes from being swept tailward [Cowley and Owen, 1989].

[17] Thus the initial merging site in the model was placed in the Southern Hemisphere, tailward of the terminator, at $(-5,8,-15)_{G S M} \mathrm{R}_{E}$ (a). Here the shear angle between the model magnetosheath and geomagnetic fields was $180^{\circ}$. The X-line was extended in both directions until the shear dropped to $70^{\circ}$ (black line); it remained tailward of the terminator along its whole length. Two sets of flux tubes are generated at the X-line; one set are generated on the southward side and move southward and tailward (solid black lines). The other set are generated on the northward side, but are also swept tailward (dashed black lines) as the model magnetosheath flow is super-Alfvénic. The FTEs on the 17 November were observed between 0250 and 0550 UT; between these times the Alfvén Mach numbers generally observed in the magnetosheath $\left(1.5<\mathrm{M}_{A}<2.5\right)$ compare well with the values predicted by the model at the location of Cluster $\left(1.8<\mathrm{M}_{A}<2.2\right)$. Also shown in Figure 3 are the locations and velocities of the FTEs observed on the 10,12 and 17 November (grey arrows). The equatorward and duskward motion of the FTEs observed nearer the equator in both Figures 2 and 3 is consistent with the most northward dashed model FTE path. For the FTEs originating further south on the X-line, the dashed model FTE paths turn southward, which can explain the absence of equatorward-moving FTEs at higher latitudes. The FTEs observed at higher latitudes are better explained by the solid paths, which move southward and duskward. The observed velocity vectors of all of the magnetosheath FTEs on these days have components antiparallel to the observed magnetosheath magnetic field, whilst those observed in the magnetosphere/boundary layer have velocity components parallel to the local magnetic field. This is consistent with the standard polarity signatures observed in all cases.

\section{Discussion}

[18] Kawano and Russell [1997b] concluded that there were two possible mechanisms for post-terminator FTEs under strongly northward IMF conditions: a tilted equatorial $\mathrm{X}$-line, and near-cusp merging where the FTEs moved equatorward. Our observations show that the FTEs in Figures 2 and 3 are more consistent with the latter. The directions of these FTE velocities are similar to those derived from the Cooling et al. [2001] model for such a merging line and their polarities are consistent with the FTE velocities relative to the local magnetic field. In the interpretation of Kawano and Russell [1997b, Figure 10], lower latitude observations of cusp merging are a consequence of observing the lower-latitude portion of the reconnected field line. The equatorward motion in our interpretation is a consequence of super-Alfvénic flow at the X-line, as is the transient nature of merging at this site as steady-state merging cannot be supported when the magnetosheath flow 
is super-Alfvénic [Cowley and Owen, 1989]. Furthermore, the Southern Hemisphere equatorward velocities observed in Figure 3 are not consistent with a tilted X-line originating at the subsolar point; if subsolar merging is forced in the model, southward flux tube motion is predicted in the vicinity of Cluster (not shown).

[19] Although the initial merging point used in the model runs was selected by requiring a $180^{\circ}$ magnetic shear, the fitting of predicted FTE paths to our observations did require the inclusion of a long X-line which extends into regions on the flank where the local shear drops down to the $70^{\circ}$ threshold of Freeman et al. [1993], implying that the FTEs were generated at a component merging site. However, in this region the simple model magnetospheric field used by Cooling et al. [2001] is less reliable than in subsolar regions.

[20] A Northern Hemisphere merging site (b) is also possible for the observed IMF. Although this site is less likely to occur due to the combined effect of the Earth's dipole tilt and the IMF $\mathrm{B}_{X}$ component corresponding to each FTE on these three days [Crooker, 1992], Cluster is not in a suitable location to determine whether (b) is active in November. In May/June, when Cluster crosses the magnetopause on the dawn flank, Northern Hemisphere merging is more likely, and FTEs will move tailward and predominantly northward. However, as can be seen from the FTE locations in Figure 1, the spacecraft crossed the magnetopause at lower latitudes in the Northern Hemisphere than in the Southern Hemisphere. Consequently the latitude of the Cluster spacecraft will generally be too low and too far upstream in the Northern Hemisphere to observe most of the FTEs which move directly down the tail, or those FTEs which do move equatorward.

[21] If the IMF $\mathrm{B}_{Y}$ component is positive and $\mathrm{B}_{Z}>0$, merging sites such as (c) and (d) are likely. Once again, Cluster's location at the Northern Hemisphere magnetopause makes observation of FTEs from site (c) less likely. However, although merging is less likely to occur in the Southern Hemisphere in May/June, Cluster is in a better position to observe the FTEs when it does. Any FTEs generated at site (d) should move either strongly southward and dawnward, or dawnward with a slight equatorward component. As with site (a), such motion should generally result in a standard polarity signature. All four FTE velocities in the dawn sector of Figure 2 are consistent with this motion, and 11 out of the 13 FTEs in Figure 1 in the southern dawn quadrant which occurred when $\mathrm{IMF} \mathrm{B}_{Y}>0$ were standard polarity events.

\section{Conclusions}

[22] We have compiled a catalogue of 446 high-latitude and flank FTEs observed by Cluster during the 2002/3 dayside magnetopause crossing season; this is the first survey of FTE velocities using four-spacecraft timing. Upstream IMF data were available for 421 of these FTEs. 120 FTEs were observed when the absolute clock angle was less than $70^{\circ}$. The locations, polarities and velocities of these FTEs are generally consistent with a long, component merging $\mathrm{X}$-line originating from a region of high magnetic shear in the lobe. However, the antiparallel merging hypothesis must be relaxed to extend merging to regions of lower shear; not all of the observed velocities would be explained by the model if the X-line were restricted to nearer $180^{\circ}$ shear. The equatorward velocities observed at lower southern latitudes (a consequence of super-Alfvénic flow at the merging site) are not consistent with an X-line centered on the subsolar point.

[23] Acknowledgments. We thank the Cluster FGM and PEACE teams, the ACE MAG and SWEPAM teams and the ACE Science Center. RCF was supported by a UK PPARC Studentship.

\section{References}

Balogh, A., et al. (2001), The Cluster magnetic field investigation: Overview of in-flight performance and initial results, Ann. Geophys., 19, $1207-1217$.

Cooling, B. M. A., C. J. Owen, and S. J. Schwartz (2001), Role of the magnetosheath flow in determining the motion of open flux tubes, J. Geophys. Res., 106, 18,763-18,776.

Cowley, S. W. H., and C. J. Owen (1989), A simple illustrative model of open flux tube motion over the dayside magnetopause, Planet. Space Sci., 37, 1461-1475.

Crooker, N. U. (1979), Dayside merging and cusp geometry, J. Geophys. Res., 84, 951-959.

Crooker, N. U. (1992), Reverse convection, J. Geophys. Res., 97, 19,36319,372 .

Daly, P. W., D. J. Williams, C. T. Russell, and E. Keppler (1981), Particle signature of magnetic flux transfer events at the magnetopause, J. Geophys. Res., 86, 1628-1632.

Dungey, J. W. (1961), Interplanetary magnetic field and the auroral zones, Phys. Rev. Lett., 6, 48-49.

Dungey, J. W. (1963), The structure of the exosphere or adventures in velocity space, in The Earth's Environment, edited by C. DeWitt, J. Hieblot, and A. Lebeau, pp. 505-550, Gordon and Breach, New York. Freeman, M. P., C. J. Farrugia, L. F. Burlaga, M. R. Hairston, M. E. Greenspan, J. M. Ruohoniemi, and R. P. Lepping (1993), The interaction of a magnetic cloud with the Earth: Ionospheric convection in the Northern and Southern hemispheres for a wide range of quasi-steady interplanetary magnetic field conditions, J. Geophys. Res., 98, 7633-7655.

Gonzalez, W. D., and F. S. Mozer (1974), A quantitative model for the potential resulting from reconnection with an arbitrary interplanetary magnetic field, J. Geophys. Res., 79, 4186-4194.

Harvey, C. C. (1998), Spatial gradients and the volumetric tensor, in Analysis Methods for Multi-Spacecraft Data, edited by G. Paschmann and P. W. Daly, pp. 307-348, Eur. Space Agency, Paris.

Johnstone, A. D., et al. (1997), PEACE: A Plasma Electron And Current Experiment, Space Sci. Rev., 79, 351-398.

Kawano, H., and C. T. Russell (1997a), Survey of flux transfer events observed with the ISEE 1 spacecraft: Dependence on the interplanetary magnetic field, J. Geophys. Res., 102, 11,307-11,313.

Kawano, H., and C. T. Russell (1997b), Cause of postterminator flux transfer events, J. Geophys. Res., 102, 27,029-27,038.

Kuo, H., C. T. Russell, and G. Le (1995), Statistical studies of flux transfer events, J. Geophys. Res., 100, 3513-3519.

Nishida, A. (1989), Can random reconnection on the magnetopause produce the low latitude boundary layer?, Geophys. Res. Lett., 16, 227-230.

Paschmann, G., G. Haerendel, I. Papamastorakis, N. Sckopke, S. J. Bame, J. T. Gosling, and C. T. Russell (1982), Plasma and magnetic field characteristics of magnetic flux transfer events, J. Geophys. Res., 87, 21592168.

Rijnbeek, R. P., S. W. H. Cowley, D. J. Southwood, and C. T. Russell (1984), A survey of dayside flux transfer events observed by ISEE-1 and ISEE-2 magnetometers, J. Geophys. Res., 89, 786-800.

Roelof, E. C., and D. G. Sibeck (1993), Magnetopause shape as a bivariate function of interplanetary magnetic field $B_{Z}$ and solar wind dynamic pressure, J. Geophys. Res., 98, 21,421-21,450.

Russell, C. T., and R. C. Elphic (1978), Initial ISEE magnetometer results: Magnetopause observations, Space Sci. Rev., 22, 681-715.

A. N. Fazakerley, R. C. Fear, and C. J. Owen, Mullard Space Science Laboratory, University College London, Dorking RH5 6NT, UK. (rcf@mssl.ucl.ac.uk)

E. A. Lucek, Blackett Laboratory, Imperial College London, Prince Consort Road, London SW7 2BZ, UK. 